\title{
Desafios no Manuseio Peri-Operatório de Pacientes Obesos Mórbidos: Como Prevenir Complicações *
}

\section{Challenges in Perioperative Management of Morbidly Obese Patients: How to Prevent Complications}

\author{
José Otávio Costa Auler Junior, TSA ${ }^{1}$, Cindy Galvão Giannini ${ }^{2}$, Daniel Fernandes Saragiotto ${ }^{3}$
}

\section{RESUMO}

Auler Jr JOC, Giannini CG, Saragiotto DF - Desafios no Manuseio Peri-Operatório de Pacientes Obesos Mórbidos: Como Prevenir Complicações

Justificativa e Objetivos - A incidência de Obesidade Mórbida tem aumentado significativamente nas últimas décadas, especialmente nos países desenvolvidos. Os excelentes resultados observados com o tratamento cirúrgico desta condição têm feito ressurgir o interesse no manuseio anestésico deste grupo de pacientes. O objetivo deste estudo foi enfatizar pontos vitais para o anestesiologista que lida com tais pacientes.

Conteúdo - Distúrbios cardiovasculares, respiratórios, endócrinos e metabólicos são freqüentemente associados à obesidade e podem causar repercussões de significado clínico importantes no período peri-operatório nestes pacientes. Alguns aspectos de interesse prático à anestesia são discutidos nesta revisão.

Conclusões - Além da ênfase dada às complicações mais importantes e mais freqüentemente observadas e de como preveni-las, destacamos também a importância do uso do PEEP e de volumes correntes adequados, recomendando seu uso.

UNITERMOS: ANESTESIA, Geral; CIRURGIA, Geral: bariátrica; DOENÇAS: obesidade mórbida

\section{INTRODUÇÃO}

C omo citado na literatura, os excelentes resultados observados com a gastroplastia no tratamento de obesidade mórbida refratária têm feito ressurgir o interesse no manuseio anestésico deste grupo de pacientes ${ }^{1-6}$.

* Recebido da (Received from) Disciplina de Anestesiologia no Hospital das Clínicas da Faculdade de Medicina da Universidade de São Paulo, São Paulo, SP

1. Professor Titular da Disciplina de Anestesiologia do Departamento de Cirurgia da Faculdade de Medicina da Universidade de São Paulo

2. ME da Disciplina de Anestesiologia do Departamento de Cirurgia da Faculdade de Medicina da Universidade de São Paulo

3. Acadêmico do $6^{\circ}$ ano de Medicina da Faculdade de Medicina da Universidade de São Paulo

Apresentado (Submitted) em 23 de maio de 2002

Aceito (Accepted) para publicação em 26 de agosto de 2002

Correspondência para (Mail to):

Dr. José Otávio Costa Auler Junior

Instituto do Coração - Hospital das Clínicas da FMUSP

Av. Dr. Enéas de Carvalho Aguiar, 44, $2^{\circ}$ Andar

05403-000, São Paulo, SP

E-mail: auler@incor.usp.br auler@hcnet.usp.br

(c) Sociedade Brasileira de Anestesiologia, 2003

\section{SUMMARY}

Auler Jr JOC, Giannini CG, Saragiotto DF - Challenges in Perioperative Management of Morbidly Obese Patients: How to Prevent Complications

Background and Objectives - The incidence of morbid obesity has significantly increased in recent years, especially in developed countries. Excellent results of the surgical treatment of such condition have raised the interest in the anesthetic management of such patients. This study aimed at emphasizing critical issues for anesthesiologists dealing with morbidly obese patients.

Contents - Cardiovascular, respiratory, endocrine and metabolic changes have been frequently associated to obesity and may cause significant clinical repercussions in the perioperative period of such patients. Some practical anesthetic issues are discussed in this review.

Conclusions - In addition to emphasizing most significant and frequent complications and their prevention, the importance of $P E E P$ and adequate tidal volumes is also highlighted.

KEY WORDS: ANESTHESIA, General; DISEASES: morbid obesity; SURGERY, General: bariatric pórea (IMC) maior que $35 \mathrm{~kg} / \mathrm{m}^{2}$, tem aumentado significantemente nas últimas décadas, particularmente nos países desenvolvidos, e é hoje em dia uma importante causa de complicações médicas precoces e mortes prematuras ${ }^{7}$. Como a obesidade é uma condição de vida limitante, esses pacientes são freqüentemente encaminhados para cirurgias abdominais, como a gastroplastia, para tratamento da obesidade, após falha de outras modalidades terapêuticas, como dieta. A cirurgia bariátrica é considerada um método efetivo de tratamento de obesidade refratária e, de acordo o consenso da conferência de 1996 da NIH (National Institutes of Health), permanece como único tratamento eficiente de obesidade grave, principalmente para aqueles em que o tratamento clínico, a maioria deles baseados na restrição de ingestão calórica, não teve sucesso ${ }^{8}$. Contudo, o índice de mortalidade peri-operatória, descrito em pacientes com obesidade mórbida, tem sido alto $(6,6 \%)$ quando comparado ao índice de mortalidade de pacientes não obesos submetidos a cirurgias do trato gastrointestinal $(2,6 \%)^{9}$. Estes resultados podem ser explicados pelo fato de a obesidade levar a uma série de distúrbios fisiopatológicos, incluindo distúrbios cardiovasculares, respiratórios, endócrinos e metabólicos, que podem influenciar negativamente no resultado peri-operatório $4,6,10$

Vol. 53, № 2, Março - Abril, 2003 
Paralelamente a essas conseqüências clínicas, o sobrepeso pode levar a vários problemas que exigem conhecimento e perícia do anestesiologista, para serem resolvidos com sucesso. De acordo com essa idéia, existem diversas dificuldades no manuseio peri-operatório de pacientes obesos, como:

1. Escolha de um dos muitos índices existentes para correção do peso;

2. Administração das anormalidades cardíacas e respiratórias durante o período peri-operatório;

3. Conhecimento dos distúrbios metabólicos, bem como das particularidades farmacocinéticas relacionadas à obesidade, que podem causar alterações na distribuição de drogas.

O objetivo deste trabalho é apresentar alguns aspectos de interesse prático no manuseio peri-operatório de pacientes obesos, enfatizando os problemas mais freqüentes na prática e como solucioná-los.

\section{ÍNDICES DE CORREÇÃO DE PESO}

Idealmente, um índice para definir obesidade deveria ser independente de peso, massa muscular e esquelética. Sem considerar a definição de obesidade, o peso ideal obtido por diferentes métodos é também importante por várias razões, como por exemplo, na escolha da dose correta de drogas anestésicas e no ajuste do volume corrente durante a ventilação. Na literatura é possível encontrar uma série de índices para estabelecer o peso ideal; um método amplamente utilizado é o índice de massa corpórea (IMC), dado por: peso $(P)$, expresso em quilogramas dividido pela altura $(A)$, expressa em metros e elevada ao quadrado $\left(I M C=P / A^{2}\right)$. Evidentemente, este índice tenta minimizar o efeito da altura sobre o peso. São considerados valores normais de IMC entre 22 a 28 , de acordo com o país, enquanto que a obesidade mórbida é definida como valores superiores a 35. Há também a tentativa de se classificar o risco, relacionado com a obesidade, de acordo com o IMC, sendo considerados de alto risco os pacientes com valores maiores que $40^{11}$. O peso ideal também pode ser facilmente obtido por tabelas que o correlacionam com a estatura, ou simplesmente subtraindo 100 (para homens) e 105 (para mulheres) da altura do paciente expressa em centímetros ${ }^{3,5,12}$.

De acordo com a localização corpórea do tecido adiposo, a obesidade pode ser classificada em obesidade central (andróide), em que o tecido adiposo se localiza principalmente na parte superior do corpo, e obesidade periférica (ginecóide), em que a distribuição ocorre predominantemente na parte inferior do corpo, quadris, nádegas e coxas ${ }^{13}$. Como conseqüência direta deste padrão de distribuição, na obesidade andróide há maior dificuldade no acesso de vias aéreas e ventilação e, também, maior incidência de diabetes, hipertensão arterial e doença cardiovascular quando comparada à obesidade ginecóide ${ }^{14}$.

\section{ALTERAÇÕES CARDIOVASCULARES}

As alterações fisiopatológicas na função cardíaca relacionadas à obesidade são complexas. Diabetes, obesidade grave, tempo de instalação do sobrepeso, hipertensão arterial e doença coronariana podem estar associadas com disfunção cardíaca grave. Em pacientes obesos, os volumes circulatório e plasmático, débito cardíaco, pressões de enchimento e consumo de oxigênio aumentam proporcionalmente com a elevação do peso ${ }^{15}$. Um aumento do volume sangüíneo e do fluxo pulmonar predispõe os indivíduos obesos à hipertensão pulmonar, que pode ser acentuada pela vasoconstrição pulmonar relacionada a alterações pulmonares que causam hipoxemia. A síndrome da apnéia do sono, que está fortemente associada à obesidade, pode acentuar a hipoxemia em certos períodos e agravar a hipertensão pulmonar ${ }^{16}$. Falência cardíaca ocorre com freqüência em pacientes com obesidade grave e parece ser uma importante causa de morte ${ }^{15}$. Embora algum grau de insuficiência cardíaca esteja relacionado com o sobrepeso, a insuficiência cardíaca congestiva é mais comumente descrita em pacientes com ganho de peso progressivo ${ }^{17}$. Obesidade e hipertensão arterial estão intimamente associadas; certamente com o ganho de peso o sistema vascular precisa aumentar em tamanho. Arazão para a elevação da pressão arterial seria a necessidade de maior força para impulsionar o sangue através de um sistema vascular de maior extensão, em que a resistência vascular sistêmica parece se correlacionar negativamente com o ganho de peso ${ }^{18}$. Os índices de riscos para a vida, ligados à obesidade, hipertensão arterial, doença coronariana e morte súbita, aumentam progressivamente com a elevação do peso corpóreo. De acordo com estes conceitos, mesmo na ausência de sintomatologia clínica, pacientes obesos necessitam de investigação cardiovascular cuidadosa antes de qualquer cirurgia eletiva.

Alterações eletrocardiográficas são comuns em pacientes obesos, como: baixa amplitude do complexo QRS, sobrecarga atrial esquerda, sinais de hipertrofia ventricular esquerda e aumento de átrio esquerdo ${ }^{19}$. Disritmias cardíacas e defeitos na condução podem precipitar morte súbita nos pacientes obesos. Hipertrofia miocárdica, hipocalemia relacionada ao uso de diuréticos, hipoxemia e doença coronariana podem explicar a alta incidência de disritmias nesses pacientes ${ }^{20}$.

De Divitis mostrou que mesmo em pacientes jovens obesos ocorre disfunção de ventrículo esquerdo (VE), mesmo não havendo evidência clínica de cardiomiopatia ou outras doenças associadas ${ }^{15}$. A função ventricular de 16 pacientes com obesidade mórbida, candidatos a cirurgia bariátrica, sem sintomas cardíacos, foi investigada com cintilografia cardíaca pré-operatória. Neste estudo, Kral encontrou que 12 dos 16 pacientes apresentavam disfunção ventricular direita e 5 deles apresentavam dilatação ventricular direita importante. Oito dos 16 apresentavam disfunção ventricular esquerda e em 5 deles a fração de ejeção era inferior a $50 \%{ }^{17}$. Os dados de eletrocardiografia da literatura mostraram que uma maior duração da obesidade mórbida está associada com maior massa de VE, menor função sistólica de VE e diminuição do 
enchimento diastólico de VE. A indução de perda de peso leva à diminuição da massa do VE e à melhora na função sistólica e enchimento diastólico do VE ${ }^{21}$. A obesidade leva a anormalidades no relaxamento e enchimento precoces, e o enchimento diastólico é compensado pelo aumento da contribuição da contração atrial. Então, o aumento da massa do VE se associa com a diminuição progressiva do enchimento diastólico e com o aumento da pressão sistólica final de VE em pacientes com obesidade mórbida ${ }^{22}$. Em contrapartida, persiste preocupantemente a relação entre obesidade e doença arterial coronariana. Diversos estudos epidemiológicos prospectivos têm sugerido tal relação. De qualquer forma, mesmo que muitos estudos tenham sugerido a correlação entre obesidade central e doença arterial coronariana, independentemente da coexistência de fatores de risco coronarianos, como hipertensão arterial sistêmica, diabetes mellitus e hipercolesterolemia, outros estudos falharam em identificar a obesidade como um fator de risco isolado para doença arterial coronariana ${ }^{23}$.

Concluindo-se, a obesidade grave pode levar a alterações estruturais e funcionais cardíacas, mesmo na ausência de hipertensão arterial sistêmica e outras doenças coexistentes. O débito cardíaco elevado, relacionado ao aumento do volume circulatório, pode predispor à dilatação ventricular e à hipertrofia excêntrica de VE, o que resulta em disfunção diastólica, sendo que a excessiva pressão na parede pode resultar em disfunção sistólica. Pacientes obesos necessitam de investigação cardiológica cuidadosa antes do ato cirúrgico e monitorização hemodinâmica completa peri-operatória, que inclui acesso venoso central e pressão arterial invasiva. Dependendo da função cardíaca prévia, um cateter pulmonar ${ }^{4}$ e ecocardiograma transesofágico também podem ser indicados.

\section{ALTERAÇÕES RESPIRATÓRIAS}

Aobesidade impõe profundas alterações no sistema respiratório e na demanda metabólica. Obesos apresentam consumo de oxigênio e produção de dióxido de carbono aumentados, tanto em repouso quanto durante exercício físico; no entanto, a taxa metabólica basal, por ser relacionada à superfície corpórea, é geralmente normal ${ }^{24,25}$. Aatividade metabólica do tecido adiposo, o maior dispêndio energético para locomoção e o alto volume expiratório minuto, para manter a normocapnia, são explicações levantadas para justificar o consumo elevado de oxigênio. Todavia, a obesidade pode estar associada com apnéia do sono e síndrome da hipoventilação, que se acredita responsável pela redução dos volumes pulmonares, causando hipoxemia e hipercapnia ${ }^{26}$. Na posição vertical, o volume de reserva expiratório e a capacidade residual funcional (CRF) estão diminuídos, então o volume corrente pode diminuir de acordo com a capacidade de oclusão, determinando alterações da ventilação e perfusão ou mesmo shunts, com hipoxemia subseqüente. Na posição deitada, a CRF usualmente cai, agravando as trocas gasosas $^{27}$. Apesar destas mudanças nas variáveis respiratórias, os testes usuais, como a capacidade vital forçada, volume expiratório forçado no primeiro minuto e fluxo expiratório máximo são geralmente, normais na obesidade. Por outro lado, Rubisntein e col. encontraram limitação ao fluxo respiratório entre $50 \%$ e $75 \%$ da capacidade vital em pacientes obesos ${ }^{28}$. Além das alterações funcionais, pacientes obesos podem apresentar também alterações importantes de mecânica ventilatória. Há um conceito geral de que a complacência respiratória total está diminuída pelo comprometimento torácico e pulmonar, sendo o componente torácico o mais importante. A redução da complacência da parede torácica é atribuída à gordura ao redor das costelas e do tórax. Admite-se que o aumento no volume sangüíneo pulmonar é responsável pela diminuição da complacência pulmonar ${ }^{27}$. Como sabemos, o sistema respiratório é dividido em dois componentes: a caixa torácica e o pulmão. É importante definir qual desses dois componentes - ou mesmo, ambos - é responsável pelo decréscimo na complacência respiratória total em pacientes obesos sob anestesia. Aliteratura apresenta uma grande discrepância sobre tal assunto. Em pacientes acordados, as investigações, algumas vezes usando diferentes métodos, encontraram diminuição da complacência torácica ${ }^{29,30}$. Em contraposição a esses estudos, Suratt e col., comparando pacientes acordados obesos e não obesos, não encontraram nenhuma correlação entre o IMC e a complacência da parede torácica ${ }^{31}$. Por outro lado, em pacientes obesos mórbidos sedados e curarizados, quando comparados ao normal, Van Lith e col. mostraram que a complacência torácica era menor nos pacientes obesos ${ }^{32}$. O mesmo resultado foi relatado por Pelosi e col. em pacientes obesos anestesiados ${ }^{33}$. Estes autores atribuíram a redução da complacência da parede torácica ao aumento de tecido adiposo ao redor das costelas, diafragma e abdômen. Cumpre notar que os mesmos pesquisadores investigaram os efeitos do IMC sobre a mecânica ventilatória (complacência e resistência) em outro grupo de pacientes obesos anestesiados, verificando estar a redução na complacência respiratória relacionada ao aumento do IMC, causada, principalmente, pelo componente pulmonar. Neste estudo, a complacência da parede torácica foi apenas fracamente dependente do IMC, contribuindo com mínima variação da complacência pulmonar total ${ }^{34}$. Nossos estudos, em pacientes obesos mórbidos anestesiados, mostraram que o principal responsável pela diminuição da complacência respiratória poderia ser o componente pulmonar, uma vez que a complacência torácica está pouco afetada nos pacientes obesos, em comparação com os normais e não apresenta variações durante a laparotomia ${ }^{35,36}$. Estes achados reforçam a teoria do deslocamento cranial do diafragma durante a anestesia, diminuindo a CRF, complacência pulmonar e, conseqüentemente, a complacência ventilatória total.

Quando o abdômen é aberto, a pressão intra-abdominal diminui subitamente, movendo o diafragma relaxado para uma posição fora do tórax. Neste estudo, a complacência pulmonar aumentou significantemente durante o período de abertura abdominal, 48,41 $\pm 12,60$ após a indução anestésica para $72,20 \pm 18,20 \mathrm{ml} . \mathrm{cmH}_{2} \mathrm{O}^{-1}$, uma hora após a abertura do abdômen, quase $60 \%$ de aumento, diminuindo novamente 
após o fechamento do abdômen ${ }^{36}$. Hedenstierna e col. mediram a complacência torácica em 10 pacientes obesos anestesiados e determinaram que esta era normal ${ }^{37}$. Dados mais recentes de nosso estudo mostraram que obesos anestesiados, além de baixa complacência ventilatória, apresentaram aumento de resistência da via aérea, que era principalmente determinada pelo componente pulmonar. A pressão intra-abdominal pode desempenhar um papel importante na diminuição da complacência e aumento da resistência pulmonar ${ }^{35,36}$.

Outro ponto importante é a falta de consenso na literatura sobre o valor ideal de volume corrente (VC) a ser estabelecido durante a anestesia, fato este já debatido anteriormente em $\operatorname{artigos~}^{38,39}$, bem como o uso e indicação do PEEP (pressão expiratória final positiva) ${ }^{40,41}$. Como se sabe, os pacientes obesos, devido aos volumes pulmonares reduzidos, à diminuição da capacidade residual funcional e à alta capacidade de oclusão, têm tendência a desenvolver hipoxemia devido a distúrbios de ventilação/perfusão e aumento no shunt intrapulmonar $^{2,42}$. A anestesia, o relaxamento muscular e a posição deitada podem piorar esta situação. A pressão inspiratória transpulmonar adequada e/ou PEEP para manter volume final expiratório, associado a elevadas frações de oxigênio $\left(\mathrm{F}_{1} \mathrm{O}_{2}\right)$ foram recomendados para preservar a oxigenação sangüínea ${ }^{2,42}$ (Figura 1). Estudos relatam diferentes maneiras de lidar com esse problema, como altos níveis de $\mathrm{F}_{1} \mathrm{O}_{2} \mathrm{e}$ volume corrente (VC), PEEP e, até mesmo, ventilação em posição prona ${ }^{43,44}$. Em relação ao VC a ser estabelecido durante a anestesia para pacientes obesos mórbidos, a literatura enfoca três procedimentos principais: 1) volume corrente guiado pelos valores normais de $\mathrm{CO}_{2}$ expirado ${ }^{43} ; 2$ ) alto volume corrente (15 a $20 \mathrm{ml}^{\mathrm{kg}}{ }^{-1}$ ) ajustado de acordo com peso ideal calculado ${ }^{10}$; 3 ) alguns autores apenas recomendam altos níveis de VC, mas não mencionam os valores exatos $^{2,45}$. Comparando-se aos estudos que recomendam altos valores de VC, maiores que $15 \mathrm{ml} . \mathrm{kg}^{-1}$ de peso ideal, os valores de $\mathrm{VC}$ empregados em nossos pacientes obesos eram próximos de 0,62 I (calculando-se pelo peso ideal, o VC era aproximadamente $\left.11 \mathrm{ml} . \mathrm{kg}^{-1}\right)$. Pelosi e col., em estudos diferentes, empregaram valores similares de VC (média de volume corrente de 10 a $11 \mathrm{ml} \mathrm{kg}^{-1}$ de peso ideal) ${ }^{33,41}$ (Figura 2). Bardoczky e col. avaliaram o efeito de alto volume corrente (calculado pelo peso corpóreo ideal) a partir do valor basal de $13 \mathrm{ml} . \mathrm{kg}^{-1}$, com aumentos de $3 \mathrm{ml} . \mathrm{kg}^{-1}$ até se atingir o valor de $22 \mathrm{ml}_{\mathrm{kg}}{ }^{-1}$. Eles concluíram que o aumento no volume corrente não se acompanhou de um aumento importante na oxigenação e causou hipocapnia grave ${ }^{40}$. A aplicação do PEEP pode aumentar a oxigenação, embora esse achado não represente consenso na literatura ${ }^{46}$.

Eriksen e col. mostraram que em pacientes obesos, o PEEP reduzia o índice cardíaco em cerca de $20 \%$ e que aumentos similares da oxigenação poderiam ser alcançados com altos volumes correntes ${ }^{47}$. Por outro lado, Söderberg e col. observaram que o $\mathrm{PEEP}$ reduzia a $\mathrm{PaO}_{2}$ em pacientes obesos durante a anestesia ${ }^{48}$. Recentemente, Pelosi e col. relataram valores médios de $\mathrm{PaO}_{2}$ de 110,2 $\pm 29,6 \mathrm{mmHg}$ em obesos anestesiados utilizando-se $\mathrm{F}_{1} \mathrm{O}_{2}$ de $50 \%$, volume corrente

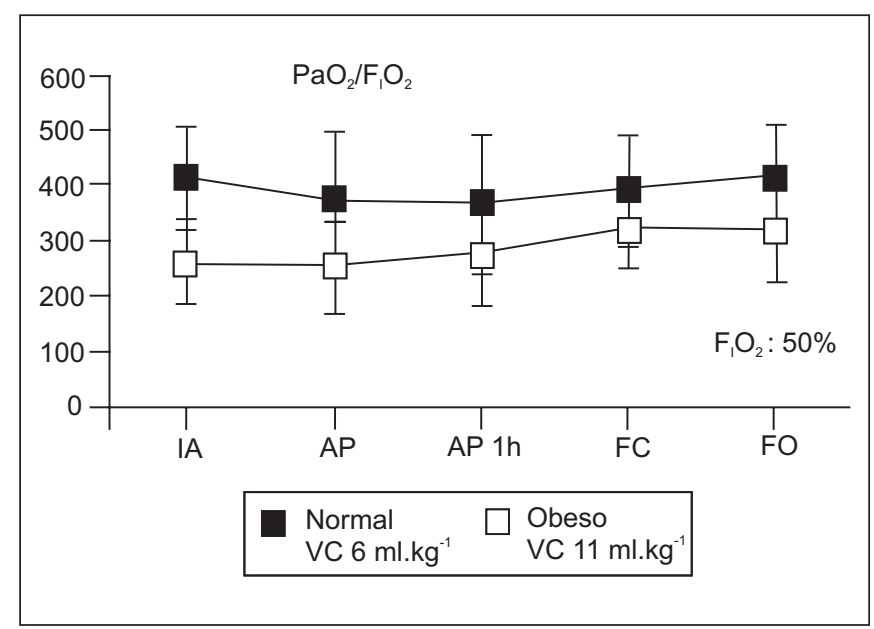

Figura 1 - Obesidade: Oxigenação durante Laparotomia Significante em: IA, AP e FO $(p<0,02)$

A relação $\mathrm{PaO}_{2} / \mathrm{FiO}_{2}$ é significativamente superior nos pacientes normais, quando comparados a obesos, durante anestesia. IA: indução da anestesia (15 min); AP: Abertura do peritônio; AP1h: abertura de peritônio após 1 h; FC: fechamento da cavidade; FO: final da operação

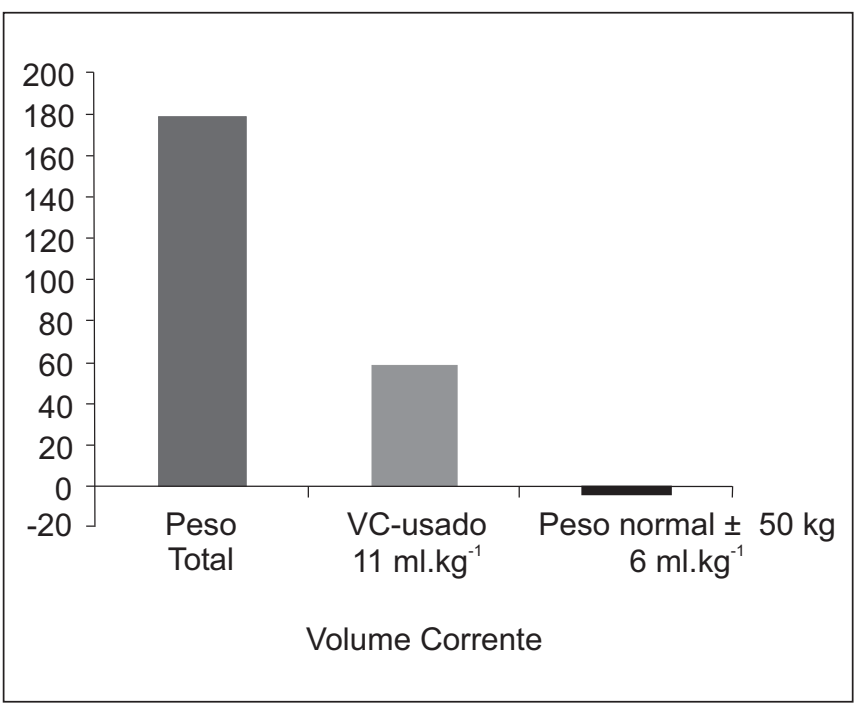

Figura 2 - \% de VC Recebido pelo Paciente Obeso

Percentual de volume corrente (VC) calculado pelo peso total, comparado ao peso ideal, em relação ao peso normal

médio de $0,683 \pm 0,043$ I. Após $10 \mathrm{cmH}_{2} \mathrm{O}$ de PEEP, eles observaram uma elevação da $\mathrm{PaO}_{2}$ de $130 \pm 28 \mathrm{mmHg}$. De acordo com esses autores, o decréscimo na oxigenação está associado com a diminuição do volume pulmonar, sugerindo que um significante colapso pulmonar está provavelmente presente nos pacientes obesos ${ }^{41}$.

Como é sugerido pela literatura, pacientes obesos apresentam durante a anestesia uma tendência ao colapso alveolar 41. A abertura das unidades colapsadas é uma função da pressão transmural, que, de acordo com a literatura, depende de alto volume corrente não definido, que pode causar elevação importante da pressão da via aérea. Isto pode ser 
intrinsecamente prejudicial ao parênquima pulmonar. Seria, então, mais racional utilizar um volume corrente compatível com níveis aceitáveis de $\mathrm{PaO}_{2}, \mathrm{PaCO}_{2}$ e curvas $\mathrm{P}-\mathrm{V}$, para a prevenção de barotrauma.

Tendo em vista tudo o que já foi discutido, recomendamos o uso do PEEP para prevenir hipoxemia, associado a volume corrente não superior a $11 \mathrm{ml}^{\mathrm{kg}}{ }^{-1}$ (peso ideal) ${ }^{36}$, de acordo com dados de mecânica respiratória.

\section{DIFICULDADES ESPERADAS NO ACESSO À VIA AÉREA}

Pacientes obesos, especialmente em decúbito dorsal horizontal, apresentam diminuição rápida da saturação arterial de oxigênio quando em apnéia, em razão da baixa reserva de oxigênio. Devido a isso, um bom planejamento da indução anestésica e intubação traqueal deve ser feito para se evitar um risco potencial de hipoxemia aguda. Pré-oxigenação em posição semi-deitada está indicada previamente à indução anestésica e intubação orotraqueal ${ }^{49}$. A literatura apresenta uma série de discrepâncias sobre este assunto. Problemas potenciais no acesso da via aérea podem ser esperados durante a anestesia em pacientes obesos mórbidos. Estes podem ser listados, começando-se por uma extensão limitada do pescoço, mandíbula e língua, que pode dificultar a visualização da laringe e da região epiglótica, ou mesmo a existência de mamas grandes, que podem interferir com o manuseio do laringoscópio. O algorritmo para via aérea difícil deve ser observado, devendo-se também incluir a inspeção cuidadosa da boca e movimentos cervicais, lâminas de laringoscópio de diferentes tamanhos, máscara laríngea e uso de intubação com o auxílio de fibroscopia devem ser considerados. Prevendo-se dificuldades para o acesso da via aérea, pode ser realizada intubação sob anestesia tópica com o paciente acordado ${ }^{50}$. Uma prática útil é olhar previamente com o uso de uma lâmina do laringoscópio dentro da boca. A região laríngea pode ser examinada após anestesia tópica, antes da indução anestésica ${ }^{51}$. A obesidade está também associada com hérnia hiatal, refluxo gastroesofágico, hiperacidez gástrica e esvaziamento gástrico diminuído. Por esses motivos, há um risco aumentado de aspiração durante a indução anestésica. Tratamento de rotina com antagonistas $\mathrm{H}_{2}$ e metoclopramida deve ser feito nestes casos ${ }^{3,5}$ (Quadro I).

Quadro I - Dificuldades Esperadas no Acesso às Vias Aéreas Inspeção cuidadosa da cavidade oral e dos movimentos cervicais Rápida diminuição da saturação arterial de $\mathrm{O}_{2}$ quando em apnéia ou DDH Movimentação limitada do pescoço e da mandíbula

Língua grande, limitando a visualização da laringe e da epiglote

Mamas volumosas e pescoço curto, interferindo com o posicionamento do laringoscópio

Maior risco de aspiração do conteúdo gástrico

Disponibilidade de lâminas de laringoscópio, máscara laríngea e estiletes de intubação em tamanhos diferentes

Fibroscopia disponível

Laringoscopia sob anestesia tópica previamente à indução

\section{ALTERAÇÕES ENDÓCRINAS, METABÓLICAS, FARMACOCINÉTICAS E FARMACODINÂMICAS}

A demanda de $\mathrm{O}_{2}$ e a produção de $\mathrm{CO}_{2}$ estão aumentadas na obesidade pelo fato de a taxa metabólica ser proporcional ao peso corpóreo. O grau de infiltração gordurosa no fígado apresenta correlação linear com o peso corpóreo, e pode estar associado com intolerância à glicose. Além do estado de tolerância aos carboidratos, a hipertrofia das ilhotas pancreáticas e a hiperinsulinemia podem refletir a alta prevalência de diabetes mellitus nos pacientes obesos ${ }^{17}$. As mudanças na distribuição das drogas nos pacientes obesos são conseqüência de modificações nos compartimentos e de aumento do débito cardíaco. A maior proporção de tecido adiposo na obesidade grave, com menor massa musculare conteúdo de água, bem como a infiltração gordurosa do fígado e a diminuição da filtração renal, podem explicar as variações no volume de distribuição, biotransformação e excreção das drogas administradas a estes pacientes ${ }^{52}$. Drogas hidrossolúveis são menos afetadas que os compostos lipofílicos em sua distribuição. Drogas lipossolúveis têm um aumento do volume de distribuição e eliminação mais prolongada. Muitas drogas são administradas considerando-se o peso total, admitindo-se que a depuração está de acordo com o peso corpóreo e o volume distribuição dos componentes por unidade de peso não se altera muito com a variação de peso. Considerando-se as mudanças nos compartimentos corpóreos, e a possível disfunção renal e hepática, estas postulações não devem ser consideradas e as doses devem ser calculadas utilizando-se índices apropriados de correção ${ }^{2}$. Pacientes obesos parecem apresentar também aumento da taxa de biotransformação de agentes anestésicos inalatórios, quando comparados a pacientes normais ${ }^{53}$. A hidratação deve pautar-se em soluções eletrolíticas balanceadas, guiando a reposição pelo peso ideal e porte da cirurgia.

Pacientes com obesidade mórbida apresentam muitas particularidades que devem ser cuidadosamente consideradas no período peri-operatório. O conhecimento da fisiopatologia da obesidade mórbida, associado ao exame de cada paciente, pode minimizar possíveis intercorrências que estão associadas ao ato anestésico-cirúrgico destes pacientes.

\section{Challenges in Perioperative Management of Morbidly Obese Patients: How to Prevent Complications}

José Otávio Costa Auler Junior, TSA, M.D., Cindy Galvão Giannini, M.D., Daniel Fernandes Saragiotto, M.D.

\section{INTRODUCTION}

Excellent results of gastroplasty to treat refractory morbid obesity have increased the interest in the anesthetic management of such patients ${ }^{1-6}$. 
Morbid obesity, defined as body mass index (BMI) above 35 $\mathrm{kg} / \mathrm{m}^{2}$, has significantly increased in recent years, especially in developed countries, and is today a major cause of early medical complications and premature death ${ }^{7}$. Since obesity is a life-limiting condition, patients are frequently referred to abdominal surgeries, such as gastroplasty, to treat obesity after other modalities, such as diet, have failed. Bariatric surgery is considered an effective method to treat refractory obesity and, according to the NIH (National Institutes of Health) 1996 conference consensus, surgery remains the only effective treatment for severe obesity, especially for those in whom clinical treatments, most of them based on caloric ingestion restrictions, have failed ${ }^{8}$. However, the perioperative mortality rate reported in morbidly obese patients is high $(6.6 \%)$ as compared to the mortality rate of non-obese patients submitted to GI tract surgeries $(2.6 \%)^{9}$. These results might be explained by the fact that obesity leads to several pathophysiological problems, including cardiovascular, respiratory, endocrine and metabolic changes which may negatively affect perioperative results ${ }^{4,6,10}$. In addition to these clinical consequences, overweight may lead to several problems requiring anesthesiologists' understanding and skill to be successfully reverted. According to this idea, there are several difficulties in perioperative management of obese patients, such as:

1. Choosing one among several weight-correction scores;

2. Managing perioperative cardiac and respiratory abnormalities;

3. Understanding obesity-related metabolic changes and pharmacokinetic particularities which may lead to drug distribution changes.

This study aimed at presenting some practical issues in the perioperative management of obese patients, emphasizing most common problems and their solutions.

\section{WEIGHT CORRECTION SCORES}

Ideally, a score to define obesity should be independent of weight, muscle and skeletal mass. Regardless of the definition of obesity, ideal weight obtained by different methods is also important for several reasons, such as accurate anesthetic drug dose and tidal volume adjustment during ventilation. There are several scores in the literature to determine optimal weight. Body mass index (BMI) is a widely used method and is determined by weight (W), expressed in kilograms, divided by height $(\mathrm{H})$ to the square, expressed in meters $\left(\mathrm{BMI}=\mathrm{W} / \mathrm{H}^{2}\right)$. It is clear that such index attempts to minimize the effect of height on weight. BMI values between 22 and 28 are considered normal, depending on the country, while morbid obesity is defined as values above 35 . There is also an attempt to classify obesity-related risks, according to $\mathrm{BMI}$, being considered of high risk patients with values above $40{ }^{11}$. Optimal weight may also be obtained through tables which correlate weight to height, or simply subtracting 100 (for males) and 105 (for females) from the height expressed in centimeters ${ }^{3,5,12}$.

According to fatty tissue location, obesity may be classified as central obesity (android), in which fatty tissue is located mainly in the upper part of the body, and peripheral obesity (gynecoid), where the distribution is predominantly in the lower part of the body, hips, breech and thighs ${ }^{13}$. As a direct consequence of this distribution pattern, it is more difficult to access airways and ventilate android obese patients; there is also a higher incidence of diabetes, arterial hypertension and cardiovascular disease as compared to gynecoid obesity ${ }^{14}$.

\section{CARDIOVASCULAR CHANGES}

Pathophysiological cardiovascular function changes related to obesity are complex. Diabetes, severe obesity, time for overweight installation, arterial hypertension and coronary disease may be associated to severe cardiac dysfunction. In obese patients, circulatory and plasma volumes, cardiac output, filling pressures and oxygen consumption increase as a function of weight gain ${ }^{15}$. Increased blood volume and pulmonary flow predisposes obese individuals to pulmonary hypertension, which may be worsened by pulmonary vasoconstriction related to pulmonary changes which lead to hypoxemia. Sleep apnea syndrome, which is strongly related to obesity, may worsen hypoxemia during certain periods thus aggravating pulmonary hypertension ${ }^{16}$. Heart failure is frequent in severely obese patients and seems to be a major cause of mortality ${ }^{15}$. Although some degree of overweight-related heart failure, congestive heart failure is more commonly described in patients with progressive weight gain 17 . Obesity and arterial hypertension are closely related; it is clear that with weight gain the vascular system has to increase in size. The reason for blood pressure increase would be the need for higher strength to push blood through a longer vascular system, where systemic vascular resistance seems to be negatively correlated to weight gain ${ }^{18}$. Life-threatening scores related to obesity, hypertension, coronary disease and sudden death progressively increase with body weight increase. According to these concepts, even in the absence of clinical symptoms, obese patients need careful cardiovascular evaluation before any elective surgery.

ECG abnormalities are common in obese patients, such as: low QRS complex amplitude, left atrial overload, signs of left ventricular hypertrophy and increased left atrium ${ }^{19}$. Arrhythmias and conduction defects may precipitate sudden death in obese patients. Myocardial hypertrophy, diureticsinduced hypokalemia, hypoxemia and coronary diseases may explain the high incidence of arrhythmias in such patients $^{20}$.

De Divitis has shown that even in young obese patients there is a left ventricle (LV) dysfunction, even without clinical evidences of cardiomyopathy or other associated diseases ${ }^{15}$. Ventricular function of 16 morbidly obese patients candidate to bariatric surgery and without cardiac symptoms, was investigated with preoperative heart scintigraphy. In this study, Kral has found that 12 out of 16 patients had right ventricular Vol. 53, N 2, Março - Abril, 2003 
dysfunction and 5 of them had major right ventricular dilatation. Eight out of 16 had left ventricular dysfunction and in 5 of them ejection fraction was below $50 \%{ }^{17}$. ECG data in the literature have shown that longer morbid obesity duration is associated to larger LV mass, lower LV systolic function and decreased LV diastolic filling. Weight loss induction leads to decreased LV mass and LV systolic function and diastolic filling improvement ${ }^{21}$. Obesity leads to early relaxation and filling abnormalities and diastolic filling is compensated by increased atrial contraction. So, increased LV mass is associated to a progressive decrease in diastolic filling and increase in LV end systolic pressure in patients with morbid obesity ${ }^{22}$. On the other hand, the relation between obesity and coronary disease persists and is of major concern. Several prospective epidemiologic studies have suggested such relation. Anyway, even with several studies suggesting a correlation between central obesity and coronary disease regardless of the coexistence of coronary risk factors, such as systemic hypertension, diabetes mellitus and hypercholesterolemia, other studies have failed to identify obesity as an isolated risk factor for coronary disease ${ }^{23}$.

Concluding, severe obesity may lead to structural and functional cardiac changes, even in the absence of systemic arterial hypertension and other coexisting diseases. High cardiac output related to increased circulatory volume may predispose to ventricular dilatation and LV eccentric hypertrophy resulting in diastolic dysfunction, and the excessive wall pressure may result in systolic dysfunction. Obese patients need careful preoperative cardiologic investigation and thorough perioperative monitoring including central venous access and invasive blood pressure. Depending on previous cardiac function, pulmonary catheter ${ }^{4}$ and transesophageal echocardiogram should also be indicated.

\section{RESPIRATORY CHANGES}

Obesity imposes deep respiratory and metabolic demand changes. Obese people have increased oxygen consumption and carbon dioxide production, both at rest and during physical exercise; baseline metabolic rate, however, for being related to body surface, is in general normal ${ }^{24,25}$. Fatty tissue metabolic function, higher energy consumption for locomotion and high expiratory minute volume to maintain normocapnia are possible explanations for high oxygen consumption. However, obesity may be associated to sleep apnea and hypoventilation syndrome, which is believed to be responsible for pulmonary volume decrease causing hypoxemia and hypercapnia ${ }^{26}$. In the upright position, expiratory reserve volume and functional residual capacity (FRC) are decreased, then tidal volume may decrease according to the occlusion capacity, determining ventilation and perfusion changes or even shunts with subsequent hypoxemia. In the horizontal position, FRC is in general decreased, worsening gas exchanges ${ }^{27}$. In spite of these changes in respiratory variables, usual tests such as forced vital capacity, forced expiratory volume in the first minute and maximum respiratory flow are in general normal in obesity. On the other hand, Rubinstein et al. have found respiratory flow limitations between $50 \%$ and $75 \%$ of vital capacity in obese patients ${ }^{28}$. In addition to functional changes, obese patients may have also major ventilatory mechanics changes. There is a general concept that total respiratory compliance is decreased by thoracic and pulmonary involvement, being the thoracic component the most important. Decreased chest wall compliance is attributed to fat around the ribs and chest. It is admitted that the increase in pulmonary blood volume is responsible for the decrease in pulmonary compliance ${ }^{27}$.

As we know, the respiratory system is divided in two components: rib cage and lungs. It is important to define which component - or even both - is responsible for the decrease in total respiratory compliance in obese patients under anesthesia. Literature is widely discrepant on the subject. In awaken patients, studies sometimes using different methods, have found a decrease in chest compliance ${ }^{29,30}$. As opposed to these studies, Suratt et al., comparing obese and non-obese awaken patients, have found no correlation between BMI and chest wall compliance ${ }^{31}$. Conversely, in sedated and curarized morbidly obese patients compared to normal patients, Van Lith et al. have shown that chest compliance was lower in obese patients ${ }^{32}$. Pelosi et al. reported the same results in anesthetized obese patients ${ }^{33}$. These authors have attributed the decrease in chest wall compliance to the increase in fatty tissue around ribs, diaphragm and abdomen. It has to be noted that the same authors have investigated BMI effects on ventilation mechanics (compliance and resistance) in a different group of anesthetized obese patients and have found that respiratory compliance related to increased BMI was mainly due to the pulmonary component. In this study, chest wall compliance was only weakly dependent on $\mathrm{BMI}$, contributing with minor total pulmonary compliance variation ${ }^{34}$. Our study with anesthetized morbidly obese patients has shown that the major responsible for respiratory compliance decrease could have been the pulmonary component, since chest compliance is poorly affected in obese patients as compared to normal patients and does not show variations during laparotomy ${ }^{35,36}$. These findings reinforce the theory of cranial diaphragm displacement during anesthesia, decreasing FRC, pulmonary compliance and, as a consequence, total ventilatory compliance.

When the abdomen is open, there is a sudden decrease in intra-abdominal pressure which displaces the relaxed diaphragm to outside the chest. In this study, pulmonary compliance has significantly increased during abdominal opening,

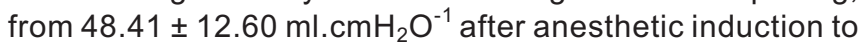
$72.20 \pm 18.20 \mathrm{ml} \mathrm{cm} \mathrm{H}_{2} \mathrm{O}^{-1}$ one hour after abdominal opening, increased almost $60 \%$, and decreasing again after the abdominal closing ${ }^{36}$. Hedestierna et al. have measured chest compliance in 10 obese anesthetized patients and have determined that it was normal ${ }^{37}$. More recent data of our study have shown that anesthetized obese patients, in addition to low ventilatory compliance, had increased airway resistance primarily determined by the pulmonary component. Intra-abdominal pressure may play an important role in decreasing compliance and increasing pulmonary resistance ${ }^{35,36}$. 
Another important point is the lack of consensus in the literature about the optimal tidal volume (TV) to be established during anesthesia, and which has already been discussed in previous studies $^{38,39}$, as well as the use and indication of PEEP (positive end expiratory pressure) ${ }^{40,41}$. As it is known, obese patients, due to decreased pulmonary volumes, decreased functional residual capacity and high occlusion capacity, tend to develop hypoxemia due to ventilation/perfusion changes and increased intrapulmonary shunt ${ }^{2,42}$. Anesthesia, muscle relaxation and horizontal position may worsen this situation. Adequate transpulmonary inspiratory pressure and/or PEEP to maintain end expiratory volume, associated to high oxygen fractions $\left(\mathrm{FiO}_{2}\right)$ have been recommended to preserve blood oxygenation ${ }^{2,42}$ (Figure 1). Studies have reported different methods to deal with this problem, such as high $\mathrm{FiO}_{2}$ and tidal volume (TV) levels, PEEP and even prone position ventilation ${ }^{43,44}$. As to TV to be determined during anesthesia for morbidly obese patients, the literature reports three major procedures: 1) tidal volume guided by normal expired $\mathrm{CO}_{2}$ values ${ }^{43} ; 2$ ) high tidal volume ( 15 to $20 \mathrm{ml} . \mathrm{kg}^{-1}$ ) adjusted according to optimal calculated weight ${ }^{10}$; 3 ) some authors recommend high TV levels without mentioning exact values ${ }^{2,45}$. As compared to studies recommending TV levels above $15 \mathrm{ml} . \mathrm{kg}^{-1}$ of optimal weight, TV values used in our study were close to $0.62 \mathrm{I}$ (calculated by optimal weight, TV was approximately $\left.11 \mathrm{ml} . \mathrm{kg}^{-1}\right)$. Pelosi et al., in different studies, have used similar TV values (tidal volume mean of 10 to $11 \mathrm{ml}^{\mathrm{kg}}{ }^{-1}$ of optimal weight) ${ }^{33,41}$ (Figure 2). Bardoczky et al. have evaluated the effect of high tidal volumes (calculated by optimal body weight) as from the baseline value of $13 \mathrm{ml} . \mathrm{kg}^{-1}$, with $3 \mathrm{ml} . \mathrm{kg}^{-1}$ increments until reaching $22 \mathrm{ml} \mathrm{kg}^{-1}$. Their conclusion was that the increase in tidal volume was not followed by a major increase in oxygenation and has caused severe hypocapnia ${ }^{40}$. PEEP may improve oxygenation although this finding is not a consensus in the literature ${ }^{46}$.

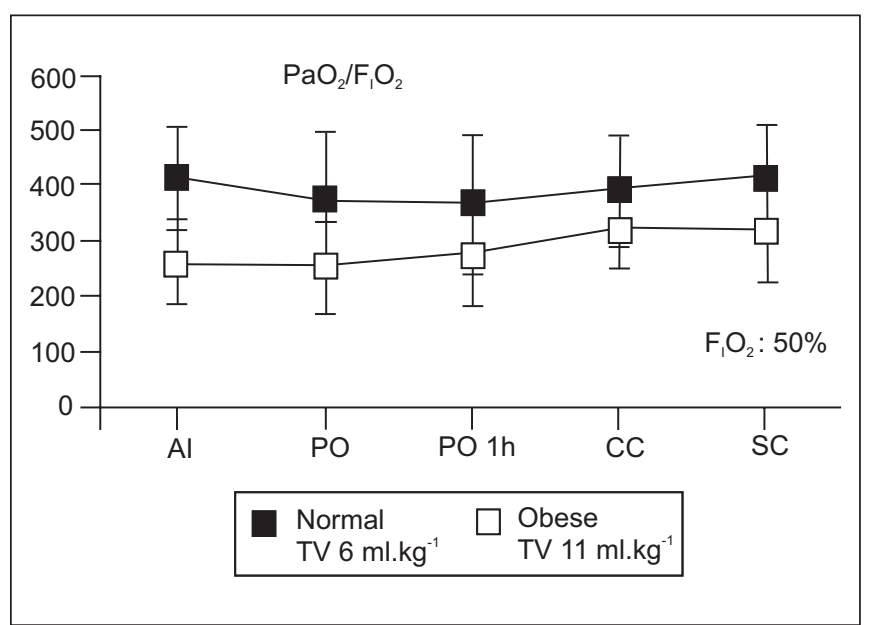

Figure 1 - Obesity: Oxygenation during Laparotomy Significant in: IA, AP and FO $(p<0.02)$

$\mathrm{PaO}_{2} / \mathrm{FiO}_{2}$ ratio is significantly higher in normal patients as compared to obese patients during anesthesia. Al: anesthetic induction (15 min); PO: peritoneal opening; PO1 h: peritoneal opening after 1 hour; CC: cavity closing; SC: surgery completion

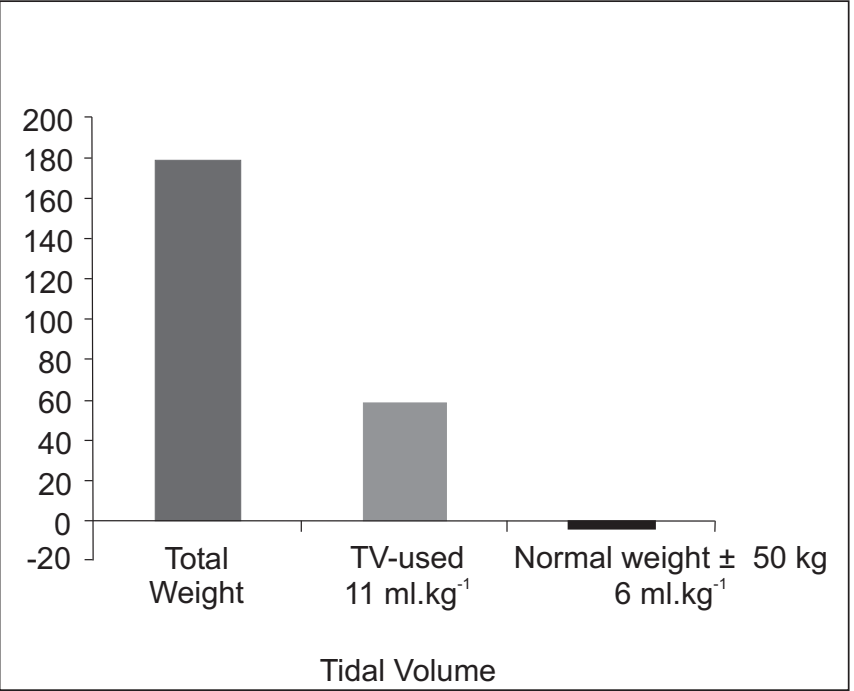

Figure 2 - \% of TV Received by Obese Patients

Tidal volume (TV) percentage calculated by total weight, by comparing optimal weight to normal weight

Eriksen et al. have shown that in obese patients, PEEP has decreased cardiac index in approximately $20 \%$ and that similar oxygenation increase could be obtained with high tidal volumes ${ }^{47}$. On the other hand, Söderberg et al. have observed that $P E E P$ has decreased $\mathrm{PaO}_{2}$ in obese patients during anesthesia ${ }^{48}$. Recently, Pelosi et al. have reported mean $\mathrm{PaO}_{2}$ values of $110.2 \pm 29.6 \mathrm{mmHg}$ in obese anesthetized patients using $\mathrm{FiO}_{2}$ equivalent to $50 \%$ of mean tidal volume of $0.683 \pm 0.043$ I. After $10 \mathrm{cmH}_{2} \mathrm{O}$ PEEP, they have observed a $\mathrm{PaO}_{2}$ increase of $130 \pm 28 \mathrm{mmHg}$. According to those authors, decreased oxygenation was associated to decreased pulmonary volume, suggesting that a significant pulmonary collapse is probably present in obese patients ${ }^{41}$.

As suggested in the literature, obese patients have a trend to alveolar collapse during anesthesia ${ }^{41}$. The opening of collapsed units is a transmural pressure function which, according to the literature, depends on high undefined tidal volumes which may cause major airway pressure increase. This may be intrinsically noxious for pulmonary parenchyma. It would be then more reasonable to use a tidal volume compatible to acceptable $\mathrm{PaO}_{2}, \mathrm{PaCO}_{2}$ and $\mathrm{P}-\mathrm{V}$ curve levels, to prevent barotraumas.

Taking into account everything that has been discussed so far, we recommend PEEP to prevent hypoxemia, associated to a tidal volume not beyond $11 \mathrm{ml}^{\mathrm{kg}} \mathrm{kg}^{-1}$ (optimal weight) ${ }^{36}$, according to respiratory mechanics data.

\section{EXPECTED DIFFICULTIES IN ACCESSING AIRWAYS}

Obese patients, especially in the supine position, show a fast oxygen arterial saturation decrease when in apnea due to low oxygen reserve. So, a good anesthetic induction and tracheal intubation plan is mandatory to prevent potential risks for acute hypoxemia. Pre-oxygenation in a semi-horizontal position is indicated before anesthetic induction and tracheal 
intubation ${ }^{49}$. Literature is discrepant about this subject. Potential airway access problems may be expected during morbidly obese patients anesthesia. These may be listed starting from a limited neck, mandible and tongue extension, which may impair larynx and epiglottis visualization, or even large breasts, which may interfere with laryngoscope handling. Difficult airway algorhythm should be observed in addition to a careful mouth and cervical movement inspection. Laryngoscope blades of different sizes, laryngeal mask and fibroscopy-aided intubation should also be considered. If airway access difficulties are expected, intubation may be performed under topic anesthesia with the patient awaken ${ }^{50}$. A good practice is to previously look inside the mouth with a laryngoscope blade. Larynx may also be examined after topic anesthesia and before anesthetic induction ${ }^{51}$. Obesity is also associated to hiatal hernia, gastroesophageal reflux, gastric hyperacidity and decreased gastric emptying. For those reasons there is an increased risk for aspiration during anesthetic induction. Routine treatment with $\mathrm{H}_{2}$ antagonists and methochlopramide should be the choice in this situations ${ }^{3,5}$ (Chart I).

Chart I - Airways Access Expected Difficulties

Carefully oral cavity and cervical movements inspection

Fast arterial $\mathrm{O}_{2}$ saturation decrease while in apnea or DDH

Limited neck and mandible movement

Large tongue limiting larynx and epiglottis visualization

Large breasts and short neck interfering with laryngoscope positioning

Higher risk for gastric content aspiration

Availability of different sizes of laryngoscope blades, laryngeal mask and intubation stilletes

Available fibroscopy

Laryngoscopy under topic anesthesia before induction

\section{ENDOCRINE, METABOLIC, PHARMACOKINETIC AND PHARMACODYNAMIC CHANGES}

Oxygen demand and $\mathrm{CO}_{2}$ production are increased in obesity because metabolic rate is a function of body weight. Liver fatty infiltration rate has a linear correlation with body weight and may be associated to intolerance to glucose. In addition to carbohydrate tolerance, pancreatic islets hypertrophy and hyperinsulinemia may reflect the high prevalence of diabetes mellitus in obese patients ${ }^{17}$. Drug distribution changes in obese patients are a consequence of compartmental changes and increased cardiac output. A higher proportion of fatty tissue in severe obesity with lower muscle mass and water content, as well as liver fatty infiltration and renal filtration decrease, may explain variations in volume distribution, biotransformation and excretion of drugs administered to such patients ${ }^{52}$. Hydrosoluble drugs distribution is less affected than lipophylic compounds. Liposoluble drugs have increased distribution volume and slower elimination. Several drugs are administered considering total weight and admitting

Revista Brasileira de Anestesiologia

Vol. 53, № 2, Março - Abril, 2003 that total clearance is a function of body weight, and distribution volume of components per weight unit does not significantly vary with weight variation. Considering changes in body compartments and the potential renal and liver dysfunction, these assumptions should not be considered and doses should be calculated using adequate correction scores ${ }^{2}$. Obese patients also seem to have increased inhalational anesthetics biotransformation rate as compared to normal patients ${ }^{53}$. Hydration should be based on balanced electrolytic solutions and replacement should be guided by optimal weight and surgery size. Morbidly obese patients have several particularities which should be carefully considered in the perioperative period. The understanding of morbid obesity pathophysiology associated to individual patient's evaluation may minimize potential problems associated to the anesthetic-surgical procedure of such patients.

\section{REFERÊNCIAS - REFERENCES}

01. Naslund E, Backman L, Granstrom L et al - Seven year results of vertical banded gastroplasty for morbid obesity. Eur J Surg, 1997;163:281-286.

02. Buckley FP - Anesthesia for the morbidly obese patient. Can J Anaesth, 1994;41:94-100.

03. Amaral CRT, Cheibub ZB - Obesidade mórbida: implicações anestésicas. Rev Bras Anestesiol, 1991;41:273-279.

04. Braga AFA, Silva ACM, Cremonesi E - Obesidade mórbida: considerações clínicas e anestésicas. Rev Bras Anestesiol, 1999;49:201-212.

05. Lins AAA, Barbosa MSA, Brodsky JB - Anestesia para gastroplastia no paciente obeso. Rev Bras Anestesiol, 1999;49: 282-287.

06. Oliveira Filho GR, Nicolodi THC, Garcia JHS et al - Problemas clínicos pré-anestésicos de pacientes morbidamente obesos submetidos a cirurgias bariátricas: comparação com pacientes não obesos. Rev Bras Anestesiol, 2002;52:217-222.

07. McGinnis JM, Foege WH - Actual causes of death in the United States. JAMA, 1993;270:2207-2212.

08. Brolin RE - Update: NIH consensus conference. Gastrointestinal surgery for severe obesity. Nutrition, 1996;12:403-404.

09. Domínguez-Cherit G, Gonzalez R, Borunda D et al - Anesthesia for morbidly obese patients. World J Surg, 1998;22:969-973.

10. Shenkman Z, Shir Y, Brodsky JB - Perioperative management of the obese patient. Br J Anaesth, 1993;70:349-359.

11. Bray GA - Pathophysiology of obesity. Am J Clin Nutr, 1992;55: 488S-494S.

12. Harrison GG - Height-weight tables. Ann Intern Med, 1985;103: (6pt2):989-994.

13. Ashwell M, Chinn S, Stalley $S$ et al - Female fat distribution: a simple classification based on two circumference measurements. Int J Obes, 1982;6:143-152.

14. Abraham S, Johnson CL - Prevalence of severe obesity in adults in the United States. Am J Clin Nutr, 1980;33:(Suppl2):306-309.

15. De Divitis O, Fazio S, Petitto $\mathrm{M}$ et al - Obesity and cardiac function. Circulation, 1981;64:477-482.

16. Laaban JP, Cassuto D, Orvoen-Frija E et al - Cardiorespiratory consequences of sleep apnea syndrome in patients with massive obesity. Eur Respir J, 1998;11:20-27.

17. Kral JG - Morbid obesity and related health risks. Ann Intern Med, 1985;103(6pt2):1043-1047. 
18. Dustan HP - Obesity and hypertension. Ann Intern Med, 1985;103(6pt2):1047-1049.

19. Alpert MA, Terry BE, Cohen MV et al - The electrocardiogram in morbid obesity. Am J Cardiol, 2000;85:908-910.

20. Drenick EJ, Fisler JC - Sudden cardiac arrest in morbidly obese surgical patients unexplained after autopsy. Am J Surg, 1988; 155:720-726.

21. Alpert MA, Lambert CR, Panayiotou $\mathrm{H}$ et al - Relation of duration of morbid obesity to left ventricular mass, systolic function, and diastolic filling, and effect of weight loss. Am J Cardiol, 1995;76: 1194-1197.

22. Alpert MA, Lambert CR, Terry BE et al - Influence of left ventricular mass on left ventricular diastolic filling in normotensive morbid obesity. Am Heart J, 1995;130:1068-1073.

23. Alpert MA, Hashimi MW - Obesity and the heart. Am J Med Sci, 1993;306:117-123.

24. Farebrother MJB - Respiratory function and cardiorespiratory response to exercise in obesity. $\mathrm{Br} \mathrm{J}$ Dis Chest, 1979;73:211.

25. Vaughan RW - Pulmonary and Cardiovascular Derangement's in the Obese Patients, em: Brown BR - Anesthetics and the Obese Patient. Contemporary Anesthesia Pratice Series. FA Davis, Philadelphia, 1982;19.

26. Lopata M, Onal E - Mass loading, sleep apnea, and the pathogenesis of the obesity hypoventilation. Am Rev Respir Dis, 1982;126:640-645.

27. Luce JM - Respiratory complications of obesity. Chest, 1980;78:626-631.

28. Rubisntein I, Zamel N, DuBarry Let al - Airflow limitation in morbidly obese nonsmoking men. Ann Intern Med, 1990;112:828-832.

29. Sharp JT, Henry SK, Sweany WR et al - Total work of breathing in normal and obese men. J Clin Invest, 1964;43:728-739.

30. Naimark A, Cherniack RM - Compliance of the respiratory system and its components in health and obesity. J Appl Physiol, 1960;15:377-382.

31. Suratt PM, Wilhoit CS, Hsiao HS et al - Compliance of chest wall in obese subjects. J Appl Physiol, 1984;57:403-407.

32. Van Lith P, Johnson FN, Sharp JT - Respiratory elastances in relaxed and paralyzed states in normal and abnormal men. J Appl Physiol, 1967;23:475-486.

33. Pelosi P, Croci M, Ravagnan I - Total respiratory system, lung and chest wall mechanics in sedated-paralyzed postoperative morbidly obese patients. Chest, 1996;109:144-151.

34. Pelosi P, Croci M, Ravagnan I et al - The effects of body mass on lung volumes, respiratory mechanics, and gas exchange general anesthesia. Anesth Analg, 1998;87:654-660.

35. Auler Jr JOC, Miyoshi E, Fernandes CR et al - Respiratory system resistance during laparotomy in morbidly obese patients. Am J Respir Crit Care Med, 2000;161(3Pt2):A693.

36. Auler Jr JOC, Miyoshi E, Fernandes CR et al - The effects of abdominal opening on respiratory mechanics during general anesthesia in normal and morbidly obese patients: a comparative study. Anesth Analg, 2002;94:471-478.

37. Hedenstierna G, Santesson J - Breathing mechanics, dead space and gas exchanges in the extremely obese, breathing spontaneously and during anaesthesia with intermittent positive pressure ventilation. Acta Anaesthesiol Scand, 1976;20:248-254.

38. Auler Jr JOC, Fernandes CR, Miyoshi E - Anestesia para gastroplastia no paciente obeso. Rev Bras Anestesiol, 2000;50: 86.

39. Brodsky JB, Lins AAA, Barbosa MAS - Anestesia para gastroplastia no paciente obeso. Rev Bras Anestesiol, 2000;50: 87.

40. Bardoczky GI, Yernault JC, Houben JJ et al - Large tidal volume ventilation does not improve oxygenation in morbidly obese patients during anesthesia. Anesth Analg, 1995;81:385-388.
41. Pelosi P, Ravagnan I, Giurati G et al - Positive end-expiratory pressure improves respiratory function in obese but not in normal subjects during anesthesia and paralysis. Anesthesiology, 1999;91:1221-1231.

42. Öberg B, Poulsen TD - Obesity: an anaesthetic challenge. Acta Anaesthesiol Scand, 1996;40:191-200.

43. Dumont L, Mattys M, Mardirosoff C et al - Changes in pulmonary mechanics during laparoscopic gastroplasty in morbidly obese patients. Acta Anaesthesiol Scand, 1997;41:408-413.

44. Pelosi P, Croci M, Calappi E et al - Prone positioning improves pulmonary function in obese patients during general anesthesia. Anesth Analg, 1996;83:578-583.

45. Fox GS, Whalley DG, Bevan DR - Anaesthesia for the morbidly obese experience with 110 patients. Br J Anaesth, 1981;53: 811-816.

46. Salem MR, Dalal FY, Zygmunt MP et al - Does PEEP improve intraoperative arterial oxygenation in grossly obese patients? Anesthesiology, 1998;48:280-281.

47. Eriksen J, Andersen J, Rasmussen JP - Postoperative pulmonary function in obese patients after upper abdominal surgery. Acta Anaesthesiol Scand, 1977;21:336-341.

48. Söderberg M, Thomson D, White T - Respiratory, circulation and anaesthetic management in obesity. Investigation before and after jejuno-ileal bypass. Acta Anaesthesiol Scand, 1977;21: $55-61$

49. Berthoud MC, Peacock JE, Reilly CS - Effectiveness of preoxygenation in morbidly obese patients. $\mathrm{Br} \mathrm{J}$ Anesth, 1991; 67:464-466.

50. Lee JJ, Larson RH, Buckley JJ - Airway maintenance in the morbidly obese. Anesth Rev, 1980;7:33-37.

51. Buckley PF - Anesthesia and obesity and Gastrointestinal Disorders, em: Barash PG et al - Clinical Anesthesia. Philadelphia, Lippincott-Raven, 1996; 975.

52. Abernethy DR, Greenblatt DJ - Pharmacokinetics of drugs in obesity. Clin Pharmacokinet, 1982;7:108-124.

53. Higuchi $\mathrm{H}$, Satoh $\mathrm{T}$, Arimura $\mathrm{S}$ et al - Serum inorganic fluoride levels in mildly obese patients during and after sevoflurane anesthesia. Anesth Analg, 1993;77:1018-1021.

\section{RESUMEN}

Auler Jr JOC, Giannini CG, Saragiotto DF - Desafíos en el Manoseo Peri-Operatorio de Pacientes Obesos Mórbidos: Como Prevenir Complicaciones

Justificativa y Objetivos - La incidencia de Obesidad Mórbida ha aumentado significativamente en las últimas décadas, especialmente en los países desarrollados. Los excelentes resultados observados con el tratamiento quirúrgico de esta condición hizo resurgir el interés en el manoseo anestésico de este grupo de pacientes. El objetivo de este estudio fue enfatizar puntos vitales para el anestesiologista que trabaja con tales pacientes.

Contenido - Disturbios cardiovasculares, respiratorios, endocrinos y metabólicos son frecuentemente asociados a la obesidad y pueden causar repercusiones de significado clínico importantes en el período peri-operatorio en estos pacientes. Algunos aspectos de interés práctico a la anestesia son discutidos en esta revisión.

Conclusiones - Más allá de la énfasis dada a las complicaciones más importantes y más frecuentemente observadas y de como prevenirlas, destacamos también la importancia del uso del PEEP y de volúmenes corrientes adecuados, recomendando su uso. 